\title{
Hierarchical self-organization in polyelectrolyte-surfactant complexes based on heteroarm star block copolyampholytes $\dagger$
}

\author{
Matthew R. Hammond, ${ }^{a}$ Chaoxu Li, ${ }^{a}$ Constantinos Tsitsilianis ${ }^{b}$ and Raffaele Mezzenga $* a c$ \\ Received 1st October 2008, Accepted 19th November 2008 \\ First published as an Advance Article on the web 21st January 2009 \\ DOI: $10.1039 / \mathrm{b817219f}$
}

A novel heteroarm star block terpolymer bearing short polystyrene (PS) arms and an equal number of longer poly(2-vinylpyridine)-block-poly(acrylic acid) (P2VP-b-PAA) arms was used as the macromolecular template component in polyelectrolyte-surfactant supramolecular complexes. The ampholytic nature of this novel star block copolymer allowed complexation to be carried out on either the P2VP blocks (with negatively charged surfactants) or on the PAA blocks (with positively charged surfactants), depending only on the $\mathrm{pH}$ at which the complexation reaction was carried out. The various complexes displayed self-organization on both the length scale of the polyelectrolyte-surfactant complexes ( $c a$. 3.5-4 nm) and on the length scale of the overall heteroarm star block copolymer (18-39 $\mathrm{nm}$, depending on which block had been complexed). Addition of surfactants to one block versus the other results in dramatically different morphologies; when the P2VP blocks are complexed, closepacked spheres are observed, while when the PAA blocks are complexed, the material forms core-shell cylinders (PS and P2VP composing the core and shell, respectively) in a matrix of PAA(surfactant). The morphologies are discussed by combining both real-space techniques, such as transmission electron miscroscopy, and reciprocal space techniques, such as X-ray scattering at small and large angles.

\section{Introduction}

Ever since ground-breaking publications in the late 80 s and early $90 \mathrm{~s},{ }^{1-3}$ application of the concepts of supramolecular chemistry ${ }^{4,5}$ to the creation of mesomorphic polymeric materials has been an active and fruitful topic of research. The non-covalent attachment of mesogenic or non-mesogenic side-groups to a polymeric template can be accomplished via hydrogen bonding, ionic bonding, metal complexation, or combinations thereof, to cite only a few examples. ${ }^{6-9}$ The introduction of block architecture into the polymer template represented another significant step forward; the resulting "comb-coil" materials were reported to hierarchically order on two length scales: ${ }^{10,11}$ that of the polymer/ side-group complex ( $c$ a. 3-7 nm) and that of the block copolymer self-organization ${ }^{12}\left(10^{1}\right.$ to $\left.10^{2} \mathrm{~nm}\right)$.

Indeed, the supramolecular approach offers polymer scientists a broad toolbox for creating new self-organized, hierarchically ordered functional materials, ${ }^{13}$ as it affords a truly vast parameter space for material design (e.g. choice of non-covalent bonding type(s), side-groups, and macromolecular template chemistry and architecture). ${ }^{6}$ Regarding this last choice, much recent work has focused on complexes involving macromolecular

${ }^{a}$ Department of Physics and Fribourg Center for Nanomaterials, University of Fribourg, Chemin du Musée 3, 1700 Fribourg, Switzerland. E-mail: raffaele.mezzenga@unifr.ch; Fax: +41 (0)26 300 9747; Tel: +41 (0)26 3009066

${ }^{b}$ Institute of Chemical Engineering and High-Temperature Chemical Processes, ICE/HT-FORTH, University of Patras, P.O. Box 1414, 26504 Patras, Greece

${ }^{c}$ Nestlé Research Center, Vers-chez-les-blanc1000 Lausanne 26, Switzerland

$\dagger$ This paper is part of a Soft Matter issue highlighting the work of emerging investigators in the soft matter field. templates with even more complicated architectures, such as dendrimers, ${ }^{14,15}$ hyperbranched polymers, ${ }^{16}$ dendronized polymers, ${ }^{17-19}$ and rod-coil block copolymers. ${ }^{20-22}$ Nandan et al. have recently reported hierarchically organized materials formed by complexation of amphiphilic surfactants with heteroarm star (HAS) and block-arm star (BAS) polymers composed of polystyrene (PS) and poly(2-vinylpyridine) (PVP). ${ }^{23,24}$ The HAS had the form $\mathrm{PS}_{5} \mathrm{P}_{2} \mathrm{VP}_{5}$ and the BAS had the form $\mathrm{PS}_{5}(\mathrm{PS}-b-\mathrm{P} 2 \mathrm{VP})_{5}$, where the subscripts refer to the number of polymeric arms attached to the central star core, and results regarding morphology and order-disorder transition temperatures $\left(\mathrm{T}_{\mathrm{ODT}} \mathrm{s}\right)$ were compared with the behavior from the analogous linear PS$b$-P2VP diblock copolymer. All three polymer templates (HAS, BAS and linear) showed a lamellar morphology before complexation and a cylinder-within-lamellar morphology in the complexes. Interesting differences were found, however, in the behavior of the $\mathrm{T}_{\mathrm{ODT}}$ as a function of template architecture.

In this paper we report on ionic supramolecular complexes based upon a novel ampholytic heteroarm star (HAS) block terpolymer of the type $\mathrm{A}_{n}(\mathrm{~B}-b-\mathrm{C})_{n}$ and charged surfactants. The HAS bears about 9 PS and 9 poly(2-vinylpyridine)-block-poly(acrylic acid) (P2VP-b-PAA) arms, and is represented in Fig. 1. Recent work on such $\mathrm{PS}_{n}(\mathrm{P} 2 \mathrm{VP}-b-\mathrm{PAA})_{n}$ stars has shown a rich phase behavior in solution as a function of $\mathrm{pH}$, owing to the $\mathrm{pH}-$ dependent protonation/deprotonation equilibrium of the P2VP and PAA blocks. ${ }^{25}$ Part of the novelty of this work is in showing how one material can be complexed at one set of sites or at a different set, depending on the $\mathrm{pH}$ at which that complexation is carried out. Additionally, given the wide variety of complex mesophases predicted and observed experimentally in "simple" A-B-C triblock copolymers, ${ }^{26-28}$ the addition of a block or blocks which can be supramolecularly complexed provides even more 


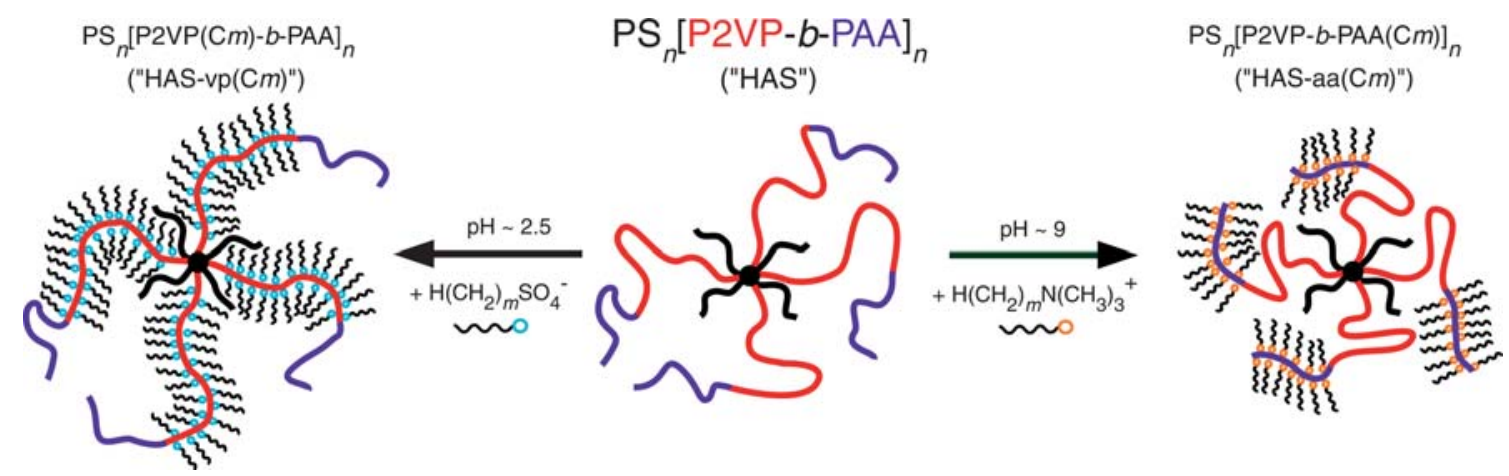

Fig. 1 Synthesis pathway for ampholytic heteroarm star terpolymer-surfactant complexes.

possibilities for material design. Details of the morphologies obtained in our HAS and HAS-based complexes, as elucidated by small- and wide-angle X-ray scattering (SAXS/WAXS) and transmission electron microscopy (TEM), are compared against previous results and predictions for uncomplexed linear and heteroarm star block copolymers, ${ }^{29}$ as well as against linear diblock comb-coil complexes. ${ }^{30}$

\section{Results and discussion}

\section{Complex preparation}

The HAS polymer used in this study was $\left(\mathrm{PS}_{33}\right)_{n}\left(\mathrm{P}_{2} \mathrm{VP}_{126}-b\right.$ $\left.\mathrm{PAA}_{69}\right)_{n}$, where the numerical subscripts denote the weight average degrees of polymerization for the corresponding block. The average arm number, $n$, was 9.2. Complexes were prepared by creating a mixture of dilute solutions of charged polymer and oppositely charged surfactants in $\mathrm{H}_{2} \mathrm{O}$ :DMSO:DMF (90:7:3 by $\mathrm{v} / \mathrm{v} / \mathrm{v}$ ), resulting in precipitation of the (neutral) complexes. ${ }^{31}$ The polymer was dissolved first in a small amount of DMSO/DMF solvent mixture and the solution diluted with water and the $\mathrm{pH}$ adjusted to either high $(\sim 9)$ or low $(\sim 2.5)$ values. At intermediate $\mathrm{pH}$ values, charged 2VP and AA groups attract and the polymer precipitates, while at the sufficiently low or high $\mathrm{pH}$ values just mentioned, only one block is charged, and the polymer remains in solution as charged micelles. ${ }^{25}$ At high $\mathrm{pH}$, complexation of the deprotonated AA groups with dodecyltrimethylammonium ( $m=12$, "C12") or octadecyltrimethylammonium ( $m=18$, "C18") yielded precipitates of $\mathrm{PS}_{n}[\mathrm{P} 2 \mathrm{VP}-b-\mathrm{PAA}(\mathrm{Cm})]_{n}$, as shown in Fig. 1 (right pathway, "HAS-aaC12" or "HAS-aaC18"). At low $\mathrm{pH}$, complexation of the protonated 2VP groups with dodecylsulfate anions yielded a precipitate of $\mathrm{PS}_{n}[\mathrm{P} 2 \mathrm{VP}(\mathrm{C} 12)-b-$ PAA $]_{n}$ (Fig. 1, left pathway, $m=12$, "HAS-vpC12"). ${ }^{32}$ The complexes, after collection, washing, and drying, proved insoluble in all common solvents as well as all mixtures thereof which might conceivably have solubilized the complexes' constituent blocks, precluding an analysis of the degree of complexation by ${ }^{1} \mathrm{H}$ NMR. Neither could the complexes be analyzed by elemental analysis, given that the atomic composition of the complexes varies only slightly from that of the parent HAS. ${ }^{33}$ However, we note that polyelectrolyte-surfactant complexes prepared by a mixture of 1:1 stoichiometry of oppositely charged groups in dilute aqueous solutions are commonly known to precipitate with preservation of the $1: 1$ stoichiometry. ${ }^{34}$ Nothing in the present experiments indicated that these complexes should be an exception. Furthermore, the resulting morphologies, as discussed below, are consistent with $100 \%$ or near- $100 \%$ complexation. Prior to characterization, all samples were solvent annealed using the procedure which is described in detail in the experimental section.

\section{Solid state morphologies of HAS and HAS-based complexes}

Table 1 summarizes the weight fractions of the various components of the HAS and the complexes that were synthesized. From the table, it is evident that complexation of one block or the other radically alters the composition. We start our discussion with results of the uncomplexed HAS material. Due to hydrogen bonding between PAA and P2VP monomers, these two blocks are miscible at room temperature. Thus we should expect, to a first approximation, the uncomplexed HAS to behave as an asymmetric $\mathrm{A}_{n} \mathrm{~B}_{n}$ heteroarm star with $f_{\mathrm{A}}=$ PS $=0.16$. Fig. 2 shows SAXS and TEM results from the uncomplexed HAS, which suggest a rather disordered array of spherical PS-rich microdomains in a matrix of P2VP/PAA. The sample section imaged in Fig. 2(a) was stained with $\mathrm{I}_{2}$ vapor, which selectively stains only 2VP groups, while Fig. 2(b) shows an image of a section stained with $\mathrm{RuO}_{4}$ vapor, which stains both PS and P2VP, but not PAA. The bright spots observed in 2(a) thus correspond to spherical PS domains (unstained) in a matrix of P2VP/PAA. The lack of good contrast in the $\mathrm{RuO}_{4}$-stained section indicates the compatibility of P2VP and PAA blocks. However, if one considers that the P2VP/PAA domains contain a lower density of $\mathrm{RuO}_{4}$-stainable groups, there should be some slight contrast; close inspection of Fig. 2(b) shows very faint spots darker than the overall matrix. The SAXS results (Fig. 2(c)) show a single peak at $q=0.41 \mathrm{~nm}^{-1}$, corresponding to a spheresphere nearest-neighbor distance of $15 \mathrm{~nm}$, which is in agreement with that observed in the TEM images. The poor ordering observed for the uncomplexed HAS is unsurprising given the large overall molecular weight of the macromolecule (Mw $199000 \mathrm{~g} / \mathrm{mol}$ in total), the complicated architecture, and the hydrogen bonding between AA and 2VP groups; these three factors greatly reduce the kinetics of polymer chain motion, necessary for the system to reach a well-ordered state.

In contrast to the very disordered spherical morphology shown in Fig. 2(a) and (b) for the uncomplexed HAS, the TEM images shown in Fig. 3(a) and (b) indicate a more organized 
Table 1 Weight fractions for various components in uncomplexed HAS or HAS-surfactant complexes

\begin{tabular}{lrllll}
\hline & \multicolumn{6}{l}{ Weight fraction } & & \\
\cline { 2 - 6 } & PS & PVP & PVP(Cm) & PAA & PAA(Cm) \\
\hline HAS (uncomplexed) & $15.7 \%$ & $60.8 \%$ & - & $23.5 \%$ & - \\
HAS-vp(C12) & $6.2 \%$ & - & $84.6 \%$ & $9.3 \%$ & - \\
HAS-aa(C12) & $9.0 \%$ & $34.9 \%$ & - & - & $56.1 \%$ \\
HAS-aa(C18) & $7.8 \%$ & $30.1 \%$ & - & - & $62.1 \%$ \\
\hline
\end{tabular}

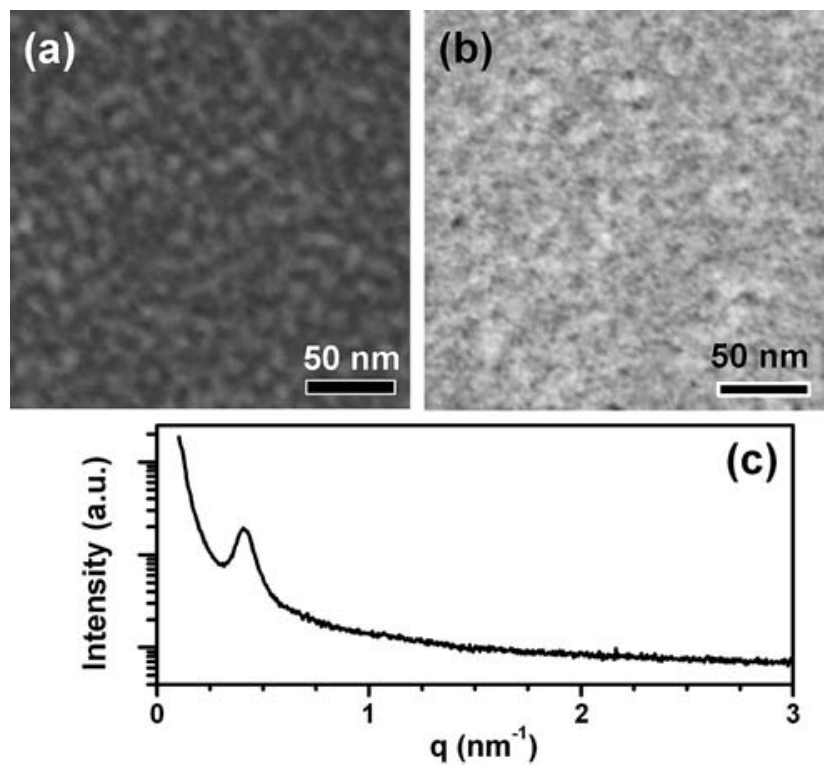

Fig. 2 (a) and (b) TEM images of the solvent annealed, uncomplexed HAS, stained with $\mathrm{I}_{2}$ (a), and $\mathrm{RuO}_{4}$ (b). (c) SAXS results for solvent annealed, uncomplexed HAS.

structure for the HAS-vp(C12) complexes. First we note that the head groups of the dodecylsulfate surfactants used in this complex contain hydrogen bond accepting groups, so one should not be surprised to see miscibility between the PAA blocks and the P2VP(C12) complex blocks. Bearing this in mind, the TEM results shown in Fig. 3(a) and (b) are consistent with miscibility of the PAA and P2VP(C12) blocks. Indeed, the $\mathrm{RuO}_{4}$-stained image, Fig. 3(a), shows dark, spherical domains in a light matrix. Similar to the case of the uncomplexed $\mathrm{HAS}, \mathrm{RuO}_{4}$ stains both P2VP and PS, but the density of stainable groups in the $\mathrm{P} 2 \mathrm{VP}(\mathrm{C} 12) / \mathrm{PAA}$ domains is dramatically lower than in the PS domains. Therefore the dark spots in Fig. 3(a) are formed by PS domains. The iodine-stained image, Fig. 3(c), shows the same spherical morphology but with the contrast reversed, i.e. light (unstained) PS domains in a matrix of stained P2VP(C12)/PAA. Again, the reduced contrast is due to the reduced density of stainable moieties in the $\mathrm{P} 2 \mathrm{VP}(\mathrm{C} 12) / \mathrm{PAA}$ domains. Because no evidence of cylinders is present in the TEM images, and considering the extremely low weight fraction of PS in this complex (6\% versus a combined $94 \%$ for the mixed $\mathrm{P} 2 \mathrm{VP}(\mathrm{C} 12) /$ PAA), a spherical morphology of PS in P2VP(C12)/PAA) is implied. Furthermore, the regular arrangements of the dark/clear
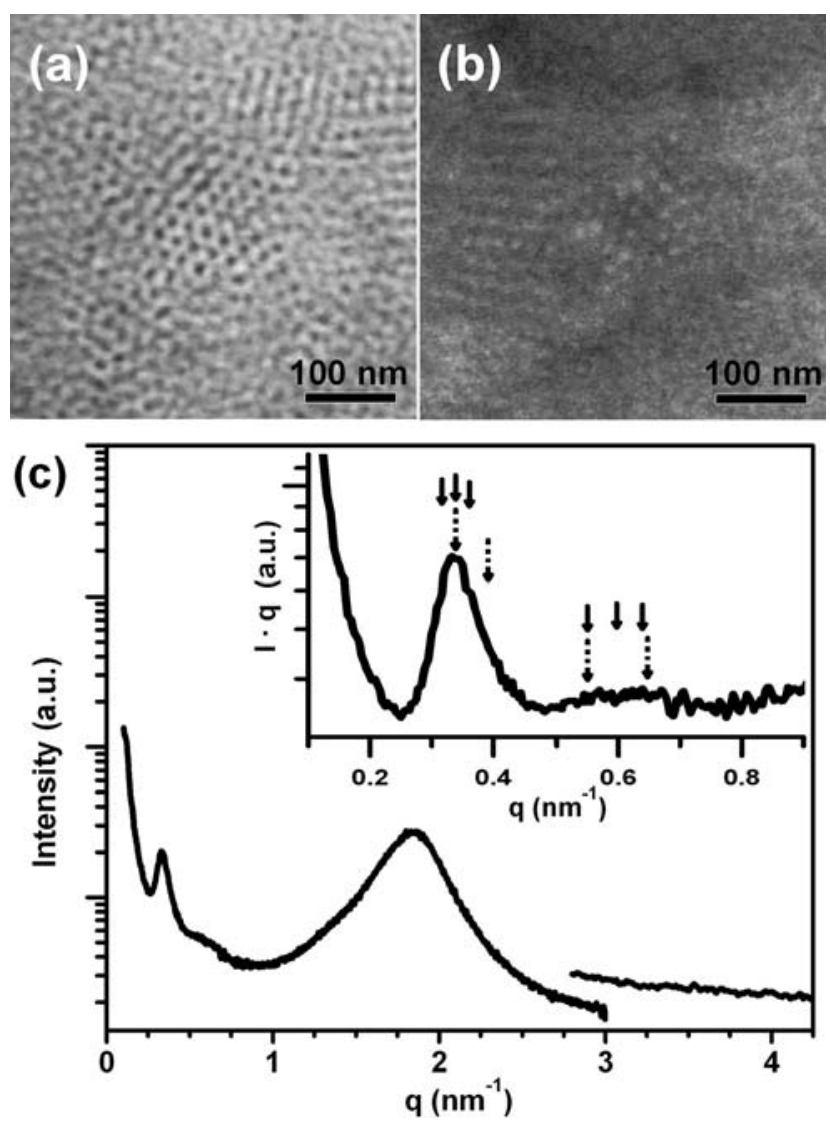

Fig. 3 (a) and (b) TEM images of sections of the HAS-vp(C12) complex stained with $\mathrm{RuO}_{4}$ (a) and with iodine (b) vapor. (c) SAXS results for the HAS-vp(C12) complex at $25^{\circ} \mathrm{C}$. Data were acquired using two different instruments, in order to provide resolution over the entire relevant $q$ range. The inset shows the data at very low angles as $I \cdot q$ vs. $q$, with arrows pointing to the positions of expected strong reflections for HCP packing (short solid arrows, from left to right: (100), (002), (101), (110), (103), (200)), and for FCC packing (longer dotted arrows, from left to right: (111), (200), (220), (311)). In the case of the HCP, the most intense reflection is expected for (002), while the predicted very weak reflection (102) is not indicated.

spots in Fig. 3(a) and (b) suggests close-packing organization of PS spheres in a regular lattice.

More insight on the lattice organization of PS spheres in this complexed HAS case can be gained by small and wide-angle X-ray scattering. Fig. 3(c) shows the SAXS profile for the HAS$\mathrm{vp}(\mathrm{C} 12)$ complex at $25^{\circ} \mathrm{C}$ : the presence of multiple peaks over a large $q$ range immediately indicates ordering on multiple length scales. The very broad peak centered at $q=1.86 \mathrm{~nm}^{-1}$ is related to ordering of the $\mathrm{P} 2 \mathrm{VP}(\mathrm{C} 12)$ complex, corresponding to a repeat distance of $3.4 \mathrm{~nm}$. The broadness of this peak and the absence of higher-order reflections preclude a definitive assignment of morphology, and are possibly to be attributed again to the $\mathrm{H}$ bonding mediated miscibility of $\mathrm{P} 2 \mathrm{VP}(\mathrm{C} 12) / \mathrm{PAA}$. The SAXS data at low- $q$, which is re-plotted as $I \cdot q$ vs. $q$ in the inset of Fig. 3(c), shows a Bragg peak at $q^{*}=0.34 \mathrm{~nm}^{-1}$ with a shoulder around $q^{*}=0.37 \mathrm{~nm}^{-1}$ and a broad bump between 0.51 and 0.72 $\mathrm{nm}^{-1}$ that may be a poorly-resolved superposition of higherorder reflections. A body-centered cubic (BCC) lattice of spheres, 
(in this space group spheres are not close-packed), can be ruled out, as this lattice would show the (200) reflection at $0.48 \mathrm{~nm}^{-1}$, which is clearly missing in the SAXS profile. On the other hand, the scattering profile is not inconsistent with hexagonal closepacked (HCP) or face-centered cubic (FCC) organization of the spheres. In the inset of Fig. 3(c) the positions of the expected reflections for HCP and FCC packing are indicated by short solid arrows and longer dashed arrows, respectively. Due to the broad multiple order reflections' halo, the SAXS profile can be considered to match either the HCP or FCC phases. We then cannot unequivocally attribute the lattice specifically to one or the other of the two phases, and consequently both HCP, FCC or coexistence thereof remain possible. Based on the above considerations, Fig. 4 sketches the molecular organization in HAS-vp(C12) complexes.

Close-packed spheres (HCP or FCC) are rarely observed in diblock copolymer melts but are nonetheless predicted by meanfield theory to exist in a very narrow region of the phase diagram at the most asymmetric compositions. ${ }^{35}$ In fact, it is remarkable that this complex exhibits microphase separation given its extremely low PS weight fraction. A couple of factors are likely to be at work here. First, ionic complexation of the P2VP block is likely to dramatically increase the value of $\chi$ between the PS and complex blocks. Valkama et al. observed spherical-withinlamellar hierarchical ordering in a hydrogen-bound PS- $b$-P4VP (pentadecylphenol) complex with a PS weight fraction as low as $8 \%{ }^{30}$ The second factor stabilizing microphase separation is related to the heteroarm star architecture; mean-field theory calculations from Olvera de la Cruz and Sanchez ${ }^{36}$ indicate that $\chi N_{\text {ODT }}$ for $\mathrm{A}_{n} \mathrm{~B}_{n}$ asymmetric heteroarm stars is significantly depressed relative to simple linear AB diblock copolymers, the effect of which increases strongly with increasing arm number, $n$.

The HAS-aa(C12) and HAS-aa(C18) complexes also show hierarchical ordering, as demonstrated by the results shown in Fig. 5. The SAXS results (Fig. 5(a)) from the HAS-aa(C18) show a Bragg peak at $q^{*}=1.77 \mathrm{~nm}^{-1}$ and a strong second-order reflection at $3.56 \mathrm{~nm}^{-1}\left(\approx 2 q^{*}\right),{ }^{37}$ indicating lamellar organization within the PAA $(\mathrm{C} 18)$ domains, with a repeat spacing of 3.5 $\mathrm{nm}$. At very low angles, a peak is observed at $0.16 \mathrm{~nm}^{-1}$, corresponding to a repeat distance at the block copolymer length scale of $39 \mathrm{~nm}$, as well as a peak at $0.45 \mathrm{~nm}^{-1}$. The latter does not correspond to a higher-order reflection of the first, and its interpretation will be discussed below with the help of real-space TEM imaging. Fig. 5(b) shows an image where the PS and PVP

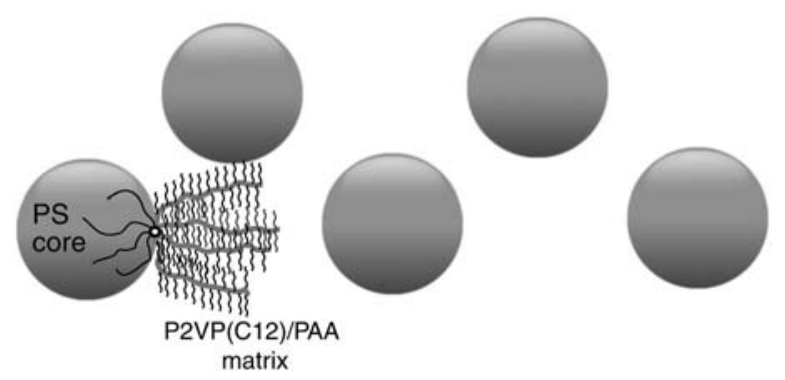

Fig. 4 Schematic drawing of the proposed morphology observed in the HAS-vp(C12) complexes. For the sake of simplicity, the PAA blocks, which are miscible with the $\mathrm{P} 2 \mathrm{VP}(\mathrm{C} 12)$ comb complex blocks, are not drawn.
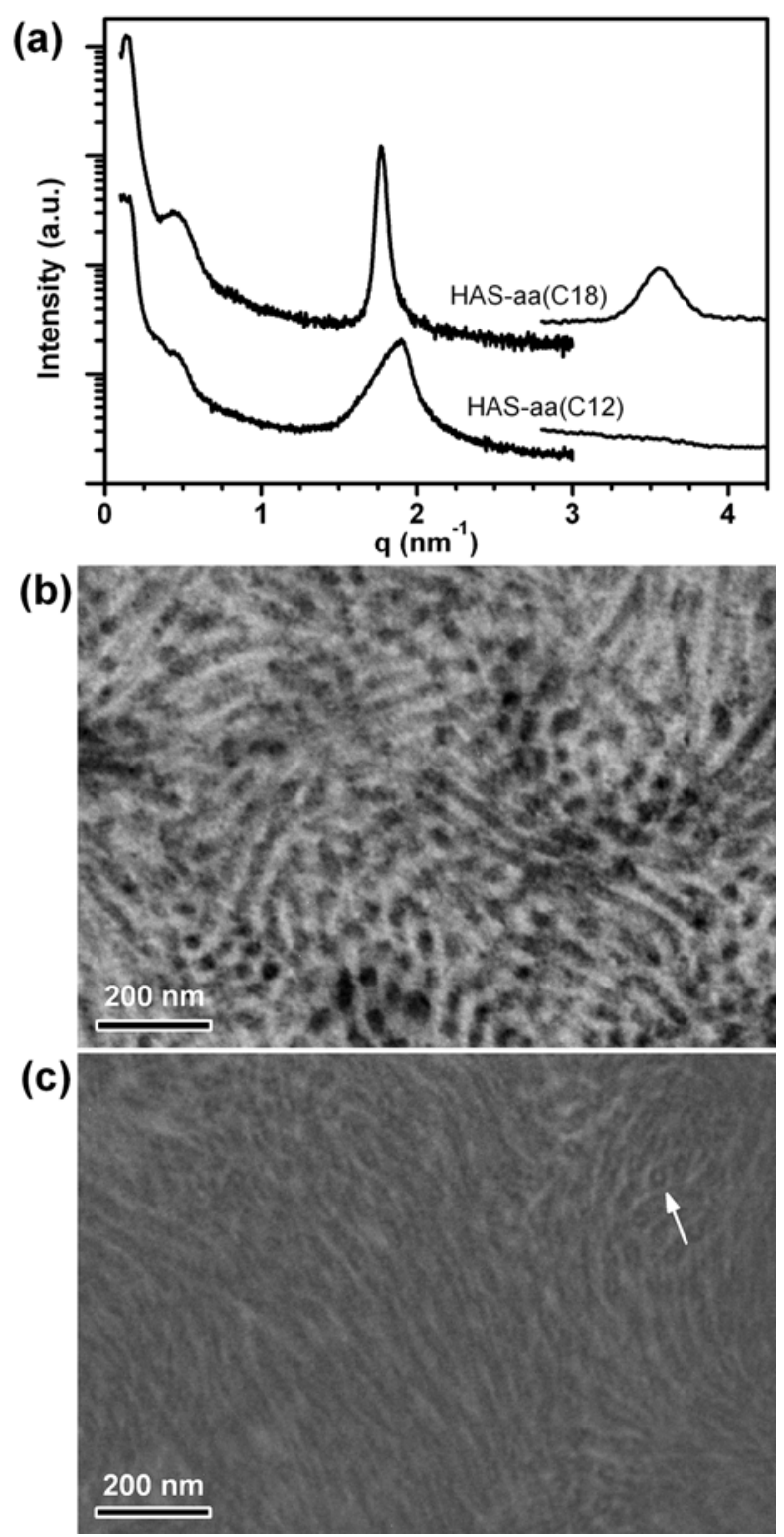

Fig. 5 (a) SAXS results for HAS-aa(Cm) complexes $(m=12,18)$ at 25 ${ }^{\circ} \mathrm{C}$. (b) and (c) TEM images of sections of the HAS-aa(C18) complex stained with $\mathrm{RuO}_{4}$ (b) and with iodine (c) vapor.

domains are stained with $\mathrm{RuO}_{4}$, demonstrating that the PS and PVP domains, together, form a rather poorly ordered array of cylinders in a matrix of (unstained) $\operatorname{PAA}(\mathrm{C} 18)$. The nearestneighbor distance measured in the image agrees well with the value of $39 \mathrm{~nm}$ measured by SAXS. After staining the P2VP domains with iodine, the image in Fig. 5(c) is obtained. The image indicates that the PS and P2VP domains form core/shell cylinders, embedded in the $\mathrm{PAA}(\mathrm{C} 18)$ matrix, a situation that is sketched in Fig. 6. Only the P2VP shell is stained, so bright regions correspond to either PS or PAA $(\mathrm{C} 18)$ domains. The white arrow in Fig. 5(c) points to a core-shell cylinder that is oriented normal to the image. We further note that the P2VP shell-to-shell spacing in Fig. 5(c) is in reasonably good agreement with the correlation distance of $14 \mathrm{~nm}$ corresponding to the 0.45 $\mathrm{nm}^{-1}$ second peak in the SAXS spectrum in Fig. 5(a). 


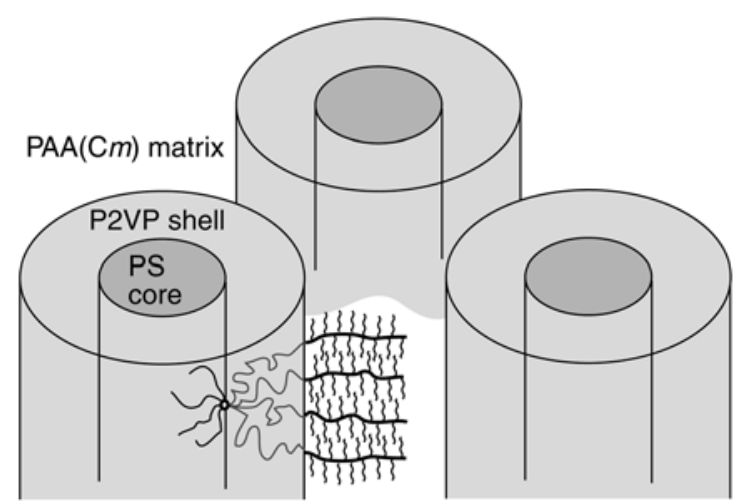

Fig. 6 Schematic drawing of the proposed core/shell cylinder-withinlamellar morphology observed in the HAS-aa $(\mathrm{Cm})$ complexes.

(a)
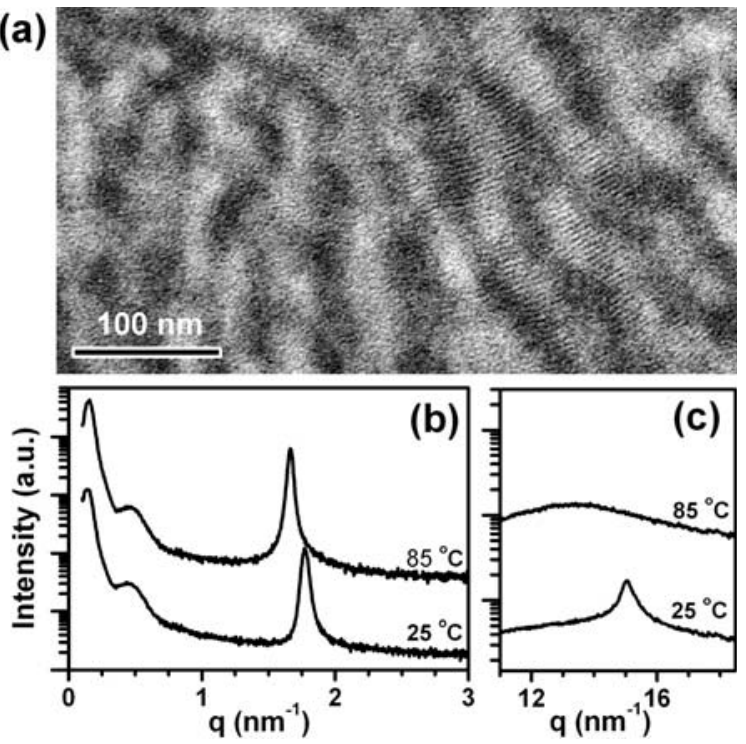

Fig. 7 (a) High magnification TEM image of a HAS-aa(C18) section stained with $\mathrm{RuO}_{4}$, showing lamellar ordering-within-the PAA(C18) complex domains. (b) SAXS and (c)WAXS data for HAS-aa(C18) complex at $25^{\circ} \mathrm{C}$ and $85^{\circ} \mathrm{C}$.

In Fig. 7(a), a higher resolution TEM image of the $\mathrm{RuO}_{4^{-}}$ stained HAS-aa(C18) sample shows the lamellar ordering within the PAA $(\mathrm{C} 18)$ complex domains. As can be observed in the right half of the image, the PAA $(\mathrm{C} 18)$ lamellae (small stripes oriented primarily horizontally) are oriented perpendicular to the PS/PVP core/shell cylinders (large scale stripes running primarily vertically), a situation that is typical for cylinder-within-lamellar coilcomb complexes ${ }^{30,38}$ and which is reflected in the schematic of Fig. 6. Again, the lamellar period observed by TEM agrees well with that measured by SAXS $(3.5 \mathrm{~nm})$. The contrast between PAA $(\mathrm{C} 18)$ lamellae in Fig. 7(a) is possible because the $\mathrm{C} 18$ surfactant tails adopt a semicrystalline configuration; the $\mathrm{RuO}_{4}$ stain diffuses more easily through the PAA(surfactant headgroup) domains than it does through the crystalline alkyl tail regions. The crystalline packing of alkyl tails at room temperature is evidenced by the peak at $15.0 \mathrm{~nm}^{-1}$ in the WAXS pattern shown in Fig. 7(c). This crystalline peak disappears upon

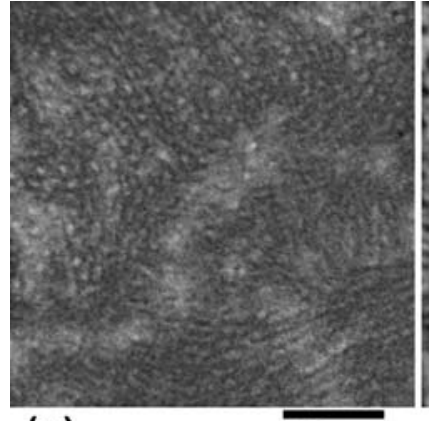

(a)
$300 \mathrm{~nm}$

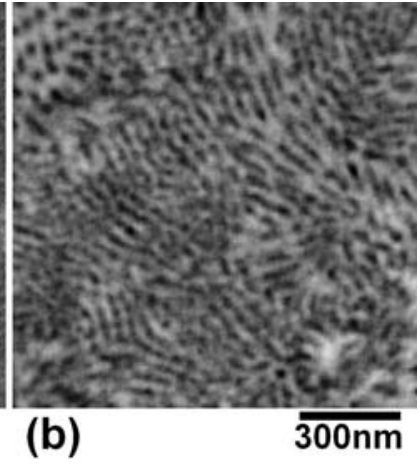

Fig. 8 TEM images of HAS-aa(C12) complex stained with iodine (a), and with $\mathrm{RuO}_{4}$ (b).

heating, as evidenced by WAXS results at $85^{\circ} \mathrm{C}$, and at that temperature the lamellar PAA $(\mathrm{C} 18)$ peak in the SAXS shifts to lower $q$, from 1.77 to $1.67 \mathrm{~nm}^{-1}$, indicating an increase in lamellar period from 3.5 to $3.8 \mathrm{~nm}$ (Fig. 7(b)). An increase of lamellar spacing upon melting of crystalline alkyl tails is to be expected. ${ }^{39}$ The melting process does not appear to change the morphology on the block copolymer length scale, as no discernable difference exists in the scattering profiles at low $q$.

The HAS-aa(C12) complex displays a hierarchically ordered morphology similar to its $\mathrm{C} 18$ analogue; TEM images of the HAS-aa(C12) complex, shown in Fig. 8, resemble those of the HAS-aa(C18) complex, and the SAXS profile (Fig. 5(a)) changes little between the two, except for a few differences. First of all, the PAA-surfactant ordering peak for the HAS-aa(C12) complex has shifted to slightly higher $q$, to $1.89 \mathrm{~nm}^{-1}$. The peak is significantly broadened, and there are no second-order peaks associated with the PAA $(\mathrm{C} 12)$ complex, implying rather poor ordering within the PAA(C12 domains) and preventing an unambiguous determination of morphology. There is no evidence in the WAXS of crystallinity of the C12 alkyl tails (data not shown). The morphology of (homopolymer) PAA(C12) complexes has been reported by Antonietti and Conrad, whose SAXS results showed a sharp primary reflection at $c a .1 .89 \mathrm{~nm}^{-1}$, and multiple higherorder reflections. ${ }^{40}$ We can only speculate that constraints imposed on PAA $(\mathrm{C} 12)$ chain packing by the complex architecture of the HAS-aaC12 prevent the PAA(C12) domains from reaching the more highly-ordered morphology observed by Antonietti and Conrad.

Concerning the scattering at low angles, the first low- $q$ peak is very slightly shifted to higher angle relative to the HAS-aa(C18) complex, to $0.17 \mathrm{~nm}^{-1}$, corresponding to a repeat distance of 37 $\mathrm{nm}$ (versus $39 \mathrm{~nm}$ for the HAS-aa(C18) complex). The peak attributed to a P2VP domain-P2VP domain correlation distance is very slightly shifted as well: $0.46 \mathrm{~nm}^{-1}$ vs. $0.45 \mathrm{~nm}^{-1}$ for the C18 analogue (13.5 vs. $14 \mathrm{~nm}$ in real-space). In summary then, the molecular arrangement of the HAS-aa(C12) complex is also consistent with the schematic representation in Fig. 6, the main difference with respect to the HAS-aa(C18) case being the amorphous packing of the $\mathrm{C} 12$ surfactant tails.

\section{Conclusions}

Hierarchically ordered polyelectrolyte-surfactant complexes were prepared from a novel asymmetric, ampholytic heteroarm 
star terpolymer, $(\mathrm{PS})_{n}(\mathrm{P} 2 \mathrm{VP}-b \text {-PAA })_{n}$, where either the polyacid (PAA) or polybase (P2VP) blocks could be complexed with oppositely charged surfactants, and their bulk morphologies were analyzed by real and reciprocal space techniques. The uncomplexed HAS material, by itself, self-organizes into spheres of PS in a mixed P2VP/PAA matrix, due to compatibility of the latter two via hydrogen bonding. In the (PS $)_{n}(\mathrm{P} 2 \mathrm{VP}(\mathrm{C} 12)-b$ $\mathrm{PAA})_{n}$ complex, the PAA blocks were also compatible with the P2VP(C12) blocks, again due to hydrogen bonding between the PAA acidic groups and the oxygens in the dodecylsulfate sulfate heads. The complex formed hierarchically ordered close-packed spheres within a matrix of mesomorphic P2VP(C12)/PAA. In contrast, the $(\mathrm{PS})_{n}(\mathrm{P} 2 \mathrm{VP}-b-\mathrm{PAA}(\mathrm{C} 12))_{n}$ and (PS) ${ }_{n}(\mathrm{P} 2 \mathrm{VP}-b$ $\mathrm{PAA}(\mathrm{C} 18))_{n}$ complexes self-assembled into arrays of core-shell cylinders in a matrix of PAA $(\mathrm{Cm})$, with the latter being itself mesomorphic (lamellar in the case of the C18 complex). This study clearly illustrates how topological design possibilities in hierarchical self-assembly of block copolymers can be greatly enhanced by increasing the level of complexity of the macromolecular templates used.

\section{Experimental}

\section{Polymer synthesis}

Complete details of the synthesis and characterization methods for the $\mathrm{PS}_{n}[\mathrm{P} 2 \mathrm{VP}-b-\mathrm{PAA}]_{n}$ heteroarm star terpolymer have recently been published elsewhere, ${ }^{25}$ but are summarized briefly here. The synthesis proceeded by a multi-step sequential "living" anionic polymerization procedure. In the first step, the polystyrene arms were prepared using s-BuLi as the initiator at -60 ${ }^{\circ} \mathrm{C}$ in THF. After the consumption of the styrene monomer a small amount of divinylbenzene (DVB) was added to the reaction medium, resulting in a star-shaped polystyrene $\left(\mathrm{PS}_{n}\right)$ precursor bearing active sites in its PDVB core. This "living" star was used to polymerize sequentially 2VP and t-butyl acrylate (tBA) at $-70{ }^{\circ} \mathrm{C}$. The final product was obtained after acidic hydrolysis of PtBA blocks. Full characterization of the HAS terpolymer was accomplished by a combination of size exclusion

Table 2 Molecular characteristics of the (PS) $)_{9}(\mathrm{P} 2 \mathrm{VP}-b-\mathrm{PAA})_{9}$ star block terpolymer

\begin{tabular}{|c|c|c|c|}
\hline Polymer & $\begin{array}{l}\text { Number } \\
\text { of arms } \\
\text { (average) }\end{array}$ & $\mathrm{M}_{\mathrm{w}}$ & $\begin{array}{l}\text { Degree of } \\
\text { polymerization }\end{array}$ \\
\hline \multicolumn{2}{|l|}{$\mathrm{PS}_{(\mathrm{arm})}$} & $\begin{array}{l}3400^{a} \\
\mathrm{M}_{\mathrm{w}} / \mathrm{M}_{\mathrm{n}}=1.1\end{array}$ & \multirow[t]{3}{*}{33} \\
\hline $\mathrm{PS}_{9 \text { (star) }}$ & 9 & $31500^{b}$ & \\
\hline $\mathrm{PS}_{9} \mathrm{P}_{2} \mathrm{VP}_{9}$ & 18 & $153000^{b}$ & \\
\hline $\begin{array}{l}\mathrm{P}^{2} \mathrm{VP}_{(\text {block, }} \text { arm) } \\
\text { (PS) }{ }_{9}(\mathrm{P} 2 \mathrm{VP}-b-\mathrm{PtBA})_{9}\end{array}$ & 18 & $\begin{array}{l}13200^{c} \\
235000^{b} \\
\mathrm{M}_{\mathrm{w}} / \mathrm{M}_{\mathrm{n}}=1.27\end{array}$ & 126 \\
\hline $\begin{array}{l}(\mathrm{PS})_{9}(\mathrm{P} 2 \mathrm{VP}-b-\mathrm{PAA})_{9} \\
\mathrm{PAA}_{(\text {block, arm })}\end{array}$ & 18 & $\begin{array}{r}199000^{d} \\
5000^{e}\end{array}$ & 69 \\
\hline \multicolumn{4}{|c|}{$\begin{array}{l}{ }^{a} \text { Determined by SEC. }{ }^{b} \text { Determined by SLS }{ }^{c} \text { Calculated by subtracting } \\
\text { the } \mathrm{M}_{\mathrm{w}} \text { of the } \mathrm{PS}_{9} \text { from that of }(\mathrm{PS})_{9} \mathrm{P} \mathrm{VP}_{9} \text { and dividing by the number of } \\
\text { arms }(9.2) .{ }^{d} \text { Calculated from the } \mathrm{M}_{\mathrm{w}} \text { of the }(\mathrm{PS})_{9}(\mathrm{P} 2 \mathrm{VP}-b-\mathrm{PtBA})_{9} \\
\text { precursor assuming quantitative hydrolysis of the tBA moieties. } \\
{ }^{e} \text { Calculated by subtracting the } \mathrm{M}_{\mathrm{w}} \text { of the } \mathrm{PS}_{9} \mathrm{P} 2 \mathrm{VP}_{9} \text { from that of } \\
(\mathrm{PS})_{9}(\mathrm{P} 2 \mathrm{VP}-b-\mathrm{PAA})_{9} \text { and dividing by the number of arms }(9.2) \text {. }\end{array}$} \\
\hline
\end{tabular}

chromatography and static light scattering, ${ }^{25}$ performed on the final product and the various intermediates, as summarized in Table 2.

\section{Polymer-surfactant complex formation}

Complexes were formed by mixing (primarily) aqueous solutions of surfactant and polymer in a 1:1 stoichiometric ratio (surfactant:charged monomer). The HAS was first dissolved in a small amount of DMSO:DMF (7:3 v/v). Dissolution required vigorous stirring and heating to $70^{\circ} \mathrm{C}$ in order to break hydrogen bonding between PAA and P2VP monomers. In the case of complexation of PAA blocks with alkyltrimethylammonium bromide (CmTAB) surfactants, 1 equivalent of $\mathrm{NaOH}$ (with respect to PAA monomers, $0.5 \mathrm{M}$ aq. solution) was added to the HAS solution, which could then be diluted with water $(\mathrm{pH} 9)$ to a final composition of (90:7:3 $\mathrm{H}_{2} \mathrm{O}$ :DMSO:DMF), and the $\mathrm{pH}$ adjusted to a final value of 9 . The final polymer concentration was $c a .0 .7$ $\mathrm{mg} / \mathrm{mL}$. Surfactant solutions were composed with the same water-DMSO-DMF compositions and adjusted to $\mathrm{pH} 9$ as well. $\mathrm{C} 12 \mathrm{TAB}$ and $\mathrm{C} 18 \mathrm{TAB}$ solutions were made with concentrations of 1.0 and $0.8 \mathrm{mg} / \mathrm{mL}$, respectively. In the case of complexation of P2VP blocks with sodium dodecylsulfate, an analogous procedure was followed, except with a target $\mathrm{pH}$ of 2.5 (i.e. addition of 1 equivalent of $\mathrm{HCl}$ with respect to $\mathrm{P} 2 \mathrm{VP}$ monomers, etc.).

Complexes were synthesized by slow addition of surfactant to polymer solutions with vigorous stirring. The precipitated complexes were collected by centrifugation, washed with water of the same $\mathrm{pH}$ as that used for the complexation, and dried thoroughly in a vacuum.

\section{Solvent annealing procedure}

Solvent annealing was performed at room temperature in a sealed container in the presence of saturated vapor of chloroform, methanol, and THF. This combination of solvents was judged to give the best combination of possibility of solubilizing all components as well as having reasonably similar vapor pressures. The samples were placed in the annealing chamber with three distinct reservoirs of solvent (one for each solvent), in order to avoid colligative changes in the saturating vapor pressures. At the end of the two days, the reservoirs were removed from the chamber and the samples allowed to dry first in the atmosphere and then in a vacuum.

\section{Small- and wide-angle X-ray scattering (SAXS, WAXS)}

X-Ray scattering experiments were performed at the SAXS beamline at the Elettra synchrotron (Trieste, Italy) with a radiation wavelength $0.154 \mathrm{~nm}$. A $1 \mathrm{D}$ gas-filled position-sensitive detector (Gabriel type) located $1.75 \mathrm{~m}$ after the sample allowed detection in the $q$ range $(q=4 \pi \sin (\theta) / \lambda$, with $2 \theta$ being the scattering angle) between $0.11<q<3.0 \mathrm{~nm}^{-1}$. Angular calibration for the small angle scattering was performed with silver behenate. ${ }^{41}$ Another, identical detector was positioned to gather wide-angle scattering data in the range $8<q<17 \mathrm{~nm}^{-1}$, with angular calibration being performed with para-bromobenzoic acid. ${ }^{42}$ For experiments where heating to $85{ }^{\circ} \mathrm{C}$ was required, a water bath heated the sample holder block, the latter being purged with helium in order to reduce any possible sample 
degradation. Data were acquired after $20 \mathrm{~min}$ of sample temperature equilibration.

In order to extend the range of measurable $q$ beyond the maximum $q=3 \mathrm{~nm}^{-1}$ obtained from the synchrotron experiments, complementary SAXS experiments were also performed using an Anton Parr SAXSess system, operated in line-collimation geometry with $\lambda=0.154 \mathrm{~nm} \mathrm{X-rays} \mathrm{and} \mathrm{a} \mathrm{sample-to-}$ detector (image plate) distance of $26 \mathrm{~cm}$.

\section{Transmission electron microscopy (TEM)}

$50 \mathrm{~nm}$ thick sections for TEM were cut using a Reichert-Jung ultramicrotome at $-60{ }^{\circ} \mathrm{C}$, deposited onto carbon-coated TEM grids and stained with either iodine or $\mathrm{RuO}_{4}$ vapor prior to analysis. Bright-field TEM images were taken using a CM100 Philips TEM operated at $80 \mathrm{kV}$. Images were acquired on a SIS Morada CCD camera.

\section{Acknowledgements}

The authors thank M. Rappolt and I. Shyjumon from the SAXS beamline at Elettra for assistance in setting up the X-ray scattering experiments. Dr Laurent Sagalowicz at Nestlé is acknowledged for useful discussions. Funding from the Swiss National Science Foundation is gratefully acknowledged.

\section{References}

1 T. Kato and J. M. J. Fréchet, Macromolecules, 1989, 22, 3818.

2 M. Antonietti, J. Conrad and A. Thünemann, Macromolecules, 1994, 27, 6007.

3 J. Ruokolainen, G. ten Brinke, O. Ikkala, M. Torkkeli and R. Serimaa, Macromolecules, 1996, 29, 3409.

4 J. M. Lehn, Angew. Chem. Int. Ed. Engl., 1988, $27,89$.

5 G. M. Whitesides, J. P. Mathias and C. T. Seto, Science, 1991, 254, 1312.

6 M. R. Hammond and R. Mezzenga, Soft Matter, 2008, 4, 952.

7 G. ten Brinke, J. Ruokolainen and O. Ikkala, Adv. Polym. Sci., 2007, 207, 113.

8 A. F. Thunemann, Prog. Polym. Sci., 2002, 27, 1473.

9 J. M. Pollino and M. Weck, Synthesis, 2002, 1277.

10 J. Ruokolainen, R. Mäkinen, M. Torkkeli, T. Mäkelä, R. Serimaa, G. ten Brinke and O. Ikkala, Science, 1998, 280, 557.

11 J. Ruokolainen, G. ten Brinke and O. Ikkala, Adv. Mater., 1999, 11, 777.

12 I. W. Hamley, The Physics of Block Copolymers, Oxford University Press, Oxford, U.K., 1998.

13 O. Ikkala and G. ten Brinke, Chem. Commun., 2004, 2131.

14 V. Percec, G. Johansson, G. Ungar and J. Zhou, J. Am. Chem. Soc., 1996, 118, 9855.

15 R. Martin-Rapun, M. Marcos, A. Omenat, J. Barbera, P. Romero and J. L. Serrano, J. Am. Chem. Soc., 2005, 127, 7397.

16 N. Canilho, M. Scholl, H.-A. Klok and R. Mezzenga, Macromolecules, 2007, 40, 8374.

17 N. Canilho, E. Kasëmi, A. D. Schlüter and R. Mezzenga, Macromolecules, 2007, 40, 2822.

18 R. Mezzenga, J. Ruokolainen, N. Canilho, E. Kasëmi, A. D. Schlüter, W. B. Lee and G. H. Fredrickson, Soft Matter, 2009, 5, 92.
19 C. Li, A. D. Schlüter, A. Zhang and R. Mezzenga, Adv. Mater., 2008, 20, 4530 .

20 S. Hanski, N. Houbenov, J. Ruokolainen, D. Chondronicola, H. Iatrou, N. Hadjichristidis and O. Ikkala, Biomacromolecules, 2006, 7, 3379.

21 M. R. Hammond, H.-A. Klok and R. Mezzenga, Macromol. Rapid Commun., 2008, 29, 299.

22 S. Hanski, S. Junnila, L. Almasy, J. Ruokolainen and O. Ikkala, Macromolecules, 2008, 41, 866.

23 B. Nandan, C.-H. Lee, H.-L. Chen and W.-C. Chen, Macromolecules, 2005, 38, 10117.

24 B. Nandan, C.-H. Lee, H.-L. Chen and W.-C. Chen, Macromolecules, 2006, 39, 4460.

25 N. Stavrouli, A. Kyriazis and C. Tsitsilianis, Macromol. Chem. Phys., 2008, 209, 2241.

26 Y. Mogi, M. Nomura, H. Kotsuji, K. Ohnishi, Y. Matsushita and I. Noda, Macromolecules, 1994, 27, 6755.

27 R. Stadler, C. Auschra, J. Beckmann, U. Krappe, I. Voight-Martin and L. Leibler, Macromolecules, 1995, 28, 3080.

28 F. S. Bates and G. H. Fredrickson, Annu. Rev. Phys. Chem., 1990, 41, 525.

29 V. Grayer, E. Dormidontova, G. Hadziioannou and C. Tsitsilianis, Macromolecules, 2000, 33, 6630.

30 S. Valkama, T. Ruotsalainen, A. Nykanen, A. Laiho, H. Kosonen, G. tenBrinke, O. Ikkala and J. Ruokolainen, Macromolecules, 2006, 39, 9327.

31 We note here that ionic polymer-surfactant complexes are often prepared by mixing acidic surfactants with polymers bearing basic groups (or vice-versa), with complexation being accomplished by proton exchange from acid to base and the ionic bonding that results. The ampholytic nature of the HAS under discussion here precludes this complexation route. On the other hand, the chosen complexation route, because it involves charging one (and only one) of the polymer blocks, avoids potential complications due to charged or hydrogen bonding interactions between the PAA and P2VP blocks.

32 Complexation at low $\mathrm{pH}$ with octadecylsulfate could not be accomplished due to low solubility of the surfactant in the DMSO/ DMF/water solvent used.

33 For example, the theoretical $\mathrm{C}, \mathrm{H}$, and $\mathrm{N}$ compositions for the parent HAS are $75 \%, 7 \%, 8 \%$, respectively, while those compositions for the HAS-aaC12 complex at $100 \%$ complexation are $77 \%, 10 \%, 7 \%$. With such slight differences in atomic composition, the value of EA as an analytical tool in this case is very limited.

34 W. J. MacKnight, E. A. Ponomarenko and D. A. Tirrell, Acc. Chem. Res., 1998, 31, 781 .

35 M. W. Matsen and F. S. Bates, Macromolecules, 1996, 29, 1091.

36 M. Olvera de la Cruz and I. C. Sanchez, Macromolecules, 1986, 19, 2501.

37 This second-order Bragg peak for the PAA(C18) lamellae is broadened relative to the first order peak due to the line-collimation of the Kratky camera setup used for the measurement at these higher $q$ values, and attendant peak smearing. See the Experimental section.

38 H.-L. Chen, J.-S. Lu, C.-H. Yu, C.-L. Yeh, U.-S. Jeng and W.C. Chen, Macromolecules, 2007, 40, 3271.

39 J. Ruokolainen, M. Torkkeli, R. Serimaa, B. E. Komanschek, O. Ikkala and G. ten Brinke, Phys. Rev. E, 1996, 54, 6646, LP.

40 M. Antonietti and J. Conrad, Angew. Chem. Int. Ed. Engl., 1994, 33, 1869.

41 T. C. Huang, H. Toraya, T. N. Blanton and Y. Wu, J. Appl. Crystallogr, 1993, 26, 180.

42 K. Ohkura, S. Kashino and M. Haisa, Bull. Chem. Soc. Japan, 1972, 45, 2651 . 